\title{
Effects of Motifs and Patterns in Decorated Fabrics on Figure Types
}

\author{
Bans - Akutey Mawufemor ${ }^{1} \quad$ Dr. Daniel Kwabena Danso ${ }^{2} \quad$ Dr. Josephine Aboagyewaa-Ntiri ${ }^{2}$ \\ 1.Department of Visual and Industrial Art, Sunyani Technical University, P. O. Box 206, Sunyani \\ 2.Department of Fashion Design and Textiles, University of Education, Winneba-Kumasi Campus, \\ P. O. Box 1277
}

\begin{abstract}
Motifs and patterns are the significant part of every culture from the ancient days. Embroidery, appliqué, tie dye/batik, prints, smocking and shirring display a rich variety of dynamic patterns and motifs in a garment. Fashion designers in Ghana appear not to take the fabric decorations into consideration when selecting and producing garments for their clients/customers. This results in the poor selection and placement of patterns and motifs in the production of garments for the various figure types. This study aimed at examining the effects of selected fabric decorations on the female figure types. The study adopted a qualitative research approach. Purposive sampling technique was used to select the twenty (20) fashion designers in Kumasi Metropolis. Structure interview was used in gather information from the fashion designers. The study discovered that that motifs and pattern decoration have effects on figure types. The effect of fabric decorations (embroidery, appliqué, tie and dye and printing, smocking and shirring) on the female figure types (Apple shape, Straight/rectangular shape, Pear shape and Hourglass shape.) helps end users to know the effects of fabric decorations on their figure type and more or less enables the designers to select and design clothes for a particular figure type in terms of choice of fabric decoration. It was recommended that fashion designers should take figure types into consideration when selecting and producing garments for their clients or end users.
\end{abstract}

Keywords: fabric, fashion designers, Apple shape, Straight/rectangular shape, Pear shape and Hourglass shape DOI: $10.7176 /$ RHSS/9-8-02

Publication date: April $30^{\text {th }} 2019$

\section{Introduction}

Fabric is a material made through weaving, knitting, spreading and crocheting used in production of garment. Cloth is a piece of fabric that has been processed. Clothes are for human body and all that goes with it enhances the individual's look. Few people have a perfect body shape. Most people improve appearance with appropriate clothing by camouflaging fewer desirable attributes and highlighting the more attractive aspects of the bodies. In order to design garments to present the best image of the wearer, it is necessary to understand the perception of beauty, and figure types as well as how the perception of body appearance can be modified through clothing (Fan, Yu \& Hunter, 2004).

According to Zheng and Jin (2014), fabrics decoration accounted for the vast majority of indoor soft adornment design, and the choice of its color controls the main hue of interior space design. In garment, print is the application of colour to the fabric in definite patterns or designs from an engraving. In properly printed fabrics, the colour is bond with the fibre, to resist washing and friction. Motifs are attractive designs or pattern, a distinctive idea in an artistic or literary composition. Motifs and patterns are the significant part of every culture form the ancient days. Embroidery, appliqué, tie dye/batik, prints, smocking and shirring display a rich variety of dynamic patterns and motifs in a garment, an observation by the researcher revealed that some tailors, seamstresses and fashion designers appear not to take the fabric decorations into consideration when selecting and producing garments for their clients/customers. This results in the poor selection and placement of patterns and motifs in the production of garments for the various figure types. This study, therefore examines the effects of selected fabric decorations on the female figure types.

\section{Literature Review}

\subsection{Motifs and Patterns}

In art, a motif is a repeated idea, pattern, image, or theme. In the textile arts, a motif is a smaller element in a much larger work. Motifs can be any size, but usually all the motifs in any given work are the same size. The patterns and stitches used in a motif may vary greatly, but there is usually some unifying element, such as texture, stitch pattern, or colour, which gives the finished piece more aesthetic appeal.

A pattern is a type of theme of recurring events or objects, sometimes referred to as elements of a set. These elements repeat in a predictable manner. It can be a template or model which can be used to generate things or parts of a thing, especially if the things that are created have enough in common for the underlying pattern to be inferred, in which case the things are said to exhibit the unique pattern. The most basic patterns based on repetition and periodicity. 


\subsection{Fabric Decoration}

Surface decoration for textiles is when one adds something to what you are making in order to make it more interesting or to add an extra bit of colour or motifs to it. It comes in all different ways and is great if you want to make something that is extra bit special. Fabric painting is a craftsmanship that began thousands of years ago in Asia and eventually expanded to other part of Asia, Europe and African through trade routes. The techniques have a deep history in Asia through Kalamkari an ancient India art, and also in China and Japan, where other forms of patterning were developed. Now recognised on a global level, fabric painting is used on a daily basis as a hobby and for business. Fabric painting has been in existence since humans began weaving fibers together to form the first fabric. As the earliest of humans painted their bodies, they began using those same techniques to paint the fabrics that they create two basic methods of fabric painting were used. Colouring was the process of directly apply an image with colour to the fabric. Patterning was printing with resist in patterns and then colouring the image on the fabric.

\subsection{Types of Fabric decorations}

Artists have been decorating fabrics since ancient times and the methods of doing so have ranged from the highly skilled to techniques that are so easy that even complete beginners can make beautiful items. Decorating fabric is a wonderful way to experiment with colours, textures and design, and the products you make can also be used in other craft projects. There are many ways to decorate fabric; among them are appliqué, printing, beads and sequins, embroidery and painting.

\subsubsection{Fabric Designing with Embroidery}

Hazel (2001) defines embroidery as the application of coloured threads onto fabric in order to enhance the fabrics appearance. Hazel further states that different threads provide different textures and patterns can range from tiny delicate designs to large areas filled with stitches. Most types of fabrics can be used for embroidery as long as the weave is even and the fabric will not be distorted by the sewing. Threads are made from silk, cotton, wool, synthetic fibres, metallic fibres, even plastics. Intricate stitches look the best and are easier to create using fine threads. Hazel again contends that few forms of decoration have had such long lasting appeal as embroidery. It is starting to realize that embroidery was carried out as long ago as $5000 \mathrm{BC}$. Today, it is still a highly fashionable way of decorating clothes and accessories.

Valerie (2005) explains that embroidery is an ancient form of needlework that has been used worldwide to embellish textiles for decorative and communicative purpose. In terms of form and aesthetics, embroidery may add colour, texture, richness and dimension. Used on clothing, it may reveal the wearer's wealth, social status, ethnic identity, or system of belief. Typically, embroidery is executed in threads of cotton, wool, silk, or linen, but may also incorporate other materials such as beads, quills, metal, shells or feathers. Some materials, techniques and stitches occur across many cultures, while others are specific to regions.

Arthur, Allen and Ingrid (2005) also state that, embroidery is the art of creating and producing raised design or motifs on woven fabrics, paper or plastics and in rarer instance, on knitted fabrics. The designs are produced with needles and threads, sometimes using a variety of colours to produce specific designs. Arthur, Allen and Ingrid (2005) continue to explained that hand embroidery is a widely practice in the past and different stitches are used to outline and fill in the design, depending upon the desired pattern.

\subsubsection{Fabric Designing with Appliqué}

According to Sorger and Jenny (2012), appliqué means to stitch one piece of fabric to another for decorative effect. Fabric motifs, such as badges can be beaded or embroidered and then appliqué onto the garment with stitch. Valerie (2005) also defines appliqué as a decorative surface design technique that adds dimensions and texture to the background fabric. The term derives from the French word "appliqué" and the Latin appliqué that means to joint or attach. While its early use was most likely to strengthen worn areas or serve as a patch over holes, appliqué developed into a creative art and used by many cultures over many centuries.

Valerie (2005) continues to say that traditional appliqué is laying pieces of fabric on fabric to form a pattern or picture. Intricate appliqué may have numerous colours and use many layers of fabrics. After each individual piece of fabric is cut out, the raw edges are turned under and hand - sewn to the background fabric using an invisible stitch. The inventions of new materials (water dissolvable stabilizer, glue sticks, and fusible web), the development of new techniques and acceptance of new standards (machine sewing verses hand sewing) have made appliqué faster and easier to do and are responsible for its continued popularity. A variation of traditional appliqué is broderie purse or chintz appliqué. Appliqué is a cut out pieces of fabric that is stitched to a larger background for decoration. An appliqué can be purchased or made by cutting out a design from one or more fabrics. Fabrics that reveals easily should not be used (Valerie 2005).

2.3.3 Fabric designing with Tie and Dye

Swati (2010) describes tie and dye as a hand process in which yarn or fabric is wrapped in certain areas with fine and strong thread. The yarn or fabric is then piece dyed and the string is removed, leaving undyed areas. The fabric is first tied and then dyed as the name implies. Different designs may be produced by knotting binding sewing by 
thread or by combination of different methods.

Tie-dye describes a pattern of colour made by preventing the dye from reaching some areas of the fabric while dyeing others. Folding, tyeing, stitching, crumpling or otherwise preparing the fabric inhibits the flow of the dye to some areas. Tie-dye is a modern term invented in the mid -1960s in the United States for a set of ancient resist - dyeing techniques, and for the products of these processes. The process of the tie -dye typically consists of folding, twisting, pleating, or crumpling fabric or a garment and binding with string or rubber bands, followed by application of dyes. Unlike traditional resist - dyeing techniques, tie - dye is characterized by the use of bright, saturated primary colours and bold patterns. This pattern, including the spiral, mandala, and peace sign and the use of multiple bold colours, has become cliched since the peak popularity of tie - dye in the 1960s and 1970s.

Picton and Mack (1991) stated that tie-and-dye-type fabrics were produced in many parts of Africa; the adire oniko cloths produced by the Yorubas of Nigeria are probably the most notable. These fabrics were produced by tying and stitching using raffia. Resist-dyed fabrics with designs composed of large or small circles were found throughout much of West Africa, and production of these was particularly common among the Yoruba's as well as the people of Senegal and Gambia. Picton and Mack (1991) continue to say that in Gambia and Senegal a marbled effect was produced by crumpling the fabric and binding it loosely before immersion in a dye bath. In Senegal, Gambia and Sierra Leone, strips of cloth were folded into several narrow pleats and bound together to create a cross-hatched effect. In Senegal, geometric patterns were embroidered on cloths which, after dyeing, were unpicked to reveal very finely rendered designs against the dyed background.

2.3.4 Fabric Design with Batik

According to Dunsmore (1996) cited by Suleiman (2012) the term batik is said to have been derived from the Malay word 'tik' which means 'to drip' or 'drop', Dunsmore (1996) but exactly where and when the technique was first practiced is uncertain. However, it is likely that the principle was discovered independently in several different areas. Moreover, (Dunsmore, 1996) further expatiated that the earliest known batiks dated to the $5^{\text {th }}-6^{\text {th }}$ Century AD, were evacuated in Egypt and include a linen cloth with white patterns showing biblical scenes blue background.

Bonifant (2009) explained that Batik dyeing was first influenced by the Chinese who used a simple form of dyeing with the use of stamps and dripping. Large pieces of fabric were stamped with a shape that was dipped in wax, and then the colour was applied, leaving the stamped areas white. Batik becomes more difficult when a second or third colour is added, and the pattern of stamps is varied. Another technique commonly used is a form of dripping where you take a paint brush and dip it in the hot wax, then let the wax drip on the cloth to make a pattern of dots. One can dip the cloth in many different colours, creating a beautiful effect. And lastly one can coat the entire cloth in wax and then roll and crack the wax, creating wavy lines throughout the fabric.

\subsubsection{Fabric Designing by Printing}

On surface treatment, Richard and Jenny (2012) are of the view that once a fabric has been constructed, it can be enhanced or altered through a range of surface treatments. Technique includes print, embellishment, dyeing and washes finisher. Richard and Jenny (2012) explain that pattern, colour and texture can be applied to fabric by printing. Fabric can be printed by various methods, including screen block, roller, mono, hand or digital printing. 2.3.6 Fabric designing with smocking

According to Debbie (2013), Smocking is a way of creating texture by marking, pinching and stitching over grids or dots to form a pattern. It's a bit like origami with fabric, and the results can be used to decorate a variety of projects, from cushion covers to clothing. Durand (1979) also stated that, smocking is an embroidery technique that is used to gather fabric using decorative threads to stitch over pleats, and provide stretch at the same time. Some kinds of smocking are worked on the right side and others are worked on the back side. In contemporary fashion, smocking is used to create elasticity at areas such as cuff and hem. Besides traditional smocking, there are various other styles of contemporary smocking. Pairs of dots are transferred on to the fabric, forming a pattern which when stitching is applied makes up a smocking design and pleats. Smocking is highly decorative but also functional on garments, which allow the panel of the garment stretch.

\subsubsection{Fabric designing with Shirring}

According to Marjorie (2007) Shirring is created by rows of stitching that is gathered on very lightweight fabrics, it is sometimes called decorative gathering. Shirring is used primarily on bodices and cuffs of garments. Colotte (1996) also explain that, Shirring is a fabric contracted into a smaller size when gathered on multiple rows of stitching sewn straight across the designated area in parallel rows. Shirring is a method of shaping a garment and is done so by controlling fullness. Its technique is similar to gathering. Shirring consists of two or more rows of gathered fabric. Shirring can be a pretty and feminine alternative to darts in small areas of a garment. Shirring can also be done on large areas of a garment like all around the top of a full skirt. Shirring works best on soft fabrics but can also done on stronger fabrics.

\section{Methodology}

The study used qualitative method and utilized primary data. The primary data was collected through field survey 
and relied on interview as a data collection instrument (Creswell, 2012). The target population for the study is the fashion designers in Kumasi Metropolis. Taking into consideration other constraints (such as financial and difficulty in selecting all the fashion designers in Kumasi) to answer the research question, 20 fashion designers in Kumasi Metropolis were purposively selected. Purposive sampling is used in qualitative research to focus on the perspectives of those who are known to experience the phenomenon of interest. All the fashion designers in Kumasi Metropolis, Ashanti Region were used as the sample frame.

The main instrument used was a structured interview guide containing both open and close-ended items. Open ended items allowed the interviewee to elaborate on their responses and also express their opinion on the subject matter.

The data gathered was analysed qualitatively to determine the findings. The data was grouped for effective management and comparison. The qualitative data analysis was consistent with interpretivist approach. This approach offers a systematic approach to collecting, organizing and analyzing data from the respondents.

\section{Results}

This section of the paper presents the result and analysis of the data obtained from the structured interview.

\subsection{Personal Details of Respondents}

In terms of the general information of the selected respondents, all 20 fashion designers responded to the questionnaire. Out of the 20 respondents who answered the questions, none of them were below 25 years, $40.0 \%$ of the respondents were between the ages of 26-35 years, and $60.0 \%$ of the respondents were between the ages of 36-45 years and none of the respondents interviewed were between the age group of 51years and above. With regards to the educational levels of respondents, none of the respondents had no formal education, $10.0 \%$ of the respondents had primary education; $35.0 \%$ had secondary education and $55.0 \%$ had tertiary education.

Concerning the years the respondents had been working as a fashion designer, $15.0 \%$ of the respondents had been working as a fashion designer for less than 5 year, $40.0 \%$ of the respondents fall between $6-10 y e a r s, 25.0 \%$ respondents said they had been working as a fashion designer for $11-15 y$ ears, and the remaining $20.0 \%$ of the respondents said that they had been a fashion designer for more than $15 y e a r s$,

\subsection{Effect of embroidery figure type}

On how embroidery affects apple shape, embroidery as the application of coloured threads on to fabric to enhance the fabric's appearance affects apple shape as a figure type. The respondents indicated that the effect depends on the size and placement of the design. They mentioned that when a large embroidery design is placed at the upper part it makes the figure look bulkier at the top and it is best at the lower part of the body and also increases the hip of the wearer when the design is done around the lower part. They further explained that, since the upper part of the body is already pronounced, the use of large embroidery would make it more pronounced and therefore should be avoided at the upper part of the body.

Concerning the rectangular shape, the fashion designers indicated that embroidery is best on the upper parts like the bust and bottom part like the hems in order to create interesting illusion for the figure and is good for the rectangular shape as the design do not have curves to break up the silhouette. They affirmed that small embroidery design have effect on the waist area when little shape is given to the body. Meanwhile, some section of the fashion designers indicated that embroidery has no effect on the rectangular shape because the shape is uniform, it can be placed at the top part as well as the lower part. They believed that embroidery looks normal on the rectangular shape and give balance to the figure shape when the design is done on upper part of the figure.

The respondents upon commenting on the effect of embroidery design on pear shape endorsed that, embroidery design affect pear shape. The respondent said the design should be at the bust level in order to conceal the defect and also to add bulkier to the upper part, so that much attention should not be placed at where the defect is but rather the attractiveness of the upper part. They emphasize that the application of coloured threads on to fabric at the lower part of the pear shape enlarge the hip section as it adds body to that area. The researcher upon further interview with the fashion designers revealed that the upper part of a pear shape is small, so the design should be bigger enough and must be arranged on the bust line in order to conform to the hips of the figure.

On the issue of embroidery's effect on hourglass shape, the respondents indicated that the hourglass shape is an ideal figure and therefore much attention should be drawn on the waist due to the size. They further mentioned that the shape can wear any fabric decoration, but to enhance the figure a broader design should be at the waist in order not to damage the figure. The design appears imbalanced when one area of the figure is made the focal point but embroidery on the hem and waist enhance balance in the figure.

\subsection{Effects of appliqué on the figure types}

The opinions of participants were sought on the effect of appliqué on the apple shape. The respondents agreed that appliqué designs have effects on the apple shape. They mentioned that appliqué is good when placed at the lower 
part of the body because the lower part is smaller, the applique make up for the big upper part. They further explained that smaller design should be used at the upper side and a little larger ones at the bottom to reduce the effect on the figure at the upper part. They added that, depending on the position of the applique it would make the figure either big or small but for the figure to be balanced it should be at the lower part to broaden or increase the width.

Touching on the effects of appliqué on rectangular shape, the respondents stated that appliqué can be placed at any part of the shape without disturbing the figure. Other respondents also believed that a bold appliqué design will be wide open for the upper and lower part of the body, a smaller appliqué is best at the waist area to give a break but larger ones at the upper and lower part of the body. They further disclosed that appliqué can be concentrated on the upper part of the body to add interest to it and draw less attention to the lower section to reveal the shape of the body.

Respondent's views were solicited on the effect of appliqué on the pear shape. The respondents opined that applique is more appropriate at the upper part of the body to create illusion and the effect that has already created at the bust line. Other respondents added that a bold appliqué design will be wide open for the upper part to add volume to it whiles a smaller appliqué will be good for the lower part.

On the hourglass, the respondents indicated that, depending on the arrangement, appliqué bring balance to the figure, emphasis should be laid at the waist area to make the figure appears more attractive. They further indicated that appliqué on the hem brings balance to the figure. Meanwhile other respondents also indicated that appliqué has got not much effect on the hourglass shape, it could be placed at the lower as well as the upper part to make the figure appears more attractive.

\subsection{Effects of Batik \& Tie-Dye on the figure types}

On the effects of tie-dyes/batik on apple shape, the respondents interviewed indicated that, depending on how the motifs in the fabrics are arranged, it can affect the balance and proportionality; larger designs should be applied at the lower part while smaller motifs at the upper part. They mentioned that smaller designs are good for the apple shape; thus, bold designs should be avoided at the upper part to draw attention away from the larger part. The respondents continue to reveal that if the designs are large then it should be better if it is placed at the lower part of the body; the addition of bold designs at the upper part should be avoided as it makes the area more bulky, illusion should be created by bringing the bold designs to the lower part.

The opinions of the fashion designers were sought for on the effects of batik/ tie-dyes on rectangular shape. The respondents agreed that, smaller or bold designs will be good for rectangular shape; the design should be all over so that the viewer's eye will not be focus at one place. The respondents still believed that larger motifs should be encouraged as it conceals the figure and add beauty to the figure because the viewers' eye will not be drawn at one area, but rather all over. On the contrary a respondent indicated a smaller design is good for rectangular shape when placed at any part of the body.

The researcher further contacted respondents to ascertain the effects of batik/ tie-dye on pear shape. The fashion designers unanimously accepted that, large motifs are good for the upper part of the body to give balance to the figure. They mentioned that since the figure is much more bulky at the lower part, smaller motifs should be used to decrease the weight and a little bigger motif at the upper part to add weight to enhance the beauty of the figure. The respondents upon further interview agreed that placing motifs and patterns at the upper part on pear shape makes the figure attractive, broader and pronounced.

On the issue of batik/ tie-dye effects on hourglass shape, the respondents opined that, the effects will depend on the motifs arrangement as the figure is an ideal type; all fabric decoration types look good on them. The respondents said that, hourglass is a balanced figure, any size be it large, medium and smaller designs will fit. Meanwhile, other respondents believed that due to its curvy form much attention should be drawn on the waist to make the figure more attractive to viewers.

\subsection{Effects of prints on figure types}

On how prints affect apple shape, the respondents indicated that prints can bring balance to the figure depending on the size and arrangement of motifs. They explained that smaller motifs are best for the upper part of the body and that large ones should be placed at the lower part since that part is small. The respondents on this issue continued to reveal that prints on apple shape bring balance when arranged in its proper part to make the figure looks attractive. It enlarges the shape when bigger prints are used at the upper part, avoid or all-over arrangement as it makes the figure look bulky said other respondents.

Concerning the rectangular shape, the respondents indicated that, depending on the type of motif(s) and design printed, it will increase or decrease the figure. They mentioned that bold prints are the best for the rectangular shape because they do not break up the figure. Smaller to medium size motifs in print fits the figure type but it should be uniform and not mixed up since the shape is almost straight. The respondents on further interview indicated that prints for garments at times create masculine touch to rectangular figure especially when smaller 
prints are used. Other respondents also said that arrangement of motifs in printed fabrics used at the waist line enhances the shape of the figure.

On the issue of prints having effects on hourglass shape, the respondents indicated that the hourglass shape is an ideal shape and the effects depend on the arrangement of the motifs. The respondents mentioned that smaller prints should be encouraged at the upper and the lower part of the body as it reduces the bulkiness of the upper and lower part, and plain at the middle to add weight to the middle. They added that most prints look good on this figure, but the busier prints make the lower part looks larger. Other respondents also revealed that both smaller and bigger-sized prints will have no effect on the body size.

\subsection{Effects of Smocking on figure types}

The opinions of respondents were sought on the effect of smocking on the apple shape. The respondents believe that smocked designs on apple shape will be good if placed at the lower parts of the dress. They mentioned that Smocking add more weight to the upper part which is already bulky, so it should be avoided at the upper part and rather placed at the waist or hip to add volume to that area. The opinions of the respondents again revealed that smocked designs on the bust line will make the figure more unbalanced, so it will be best at the lower part to make the figure balanced.

Touching on the effects of Smocking on rectangular shape, the participants stated that, smocking will add weight to the figure and should be avoided at the waist area as it will throw off balance, because the shape is uniform and it could be placed at either upper or lower part of the body. Meanwhile other respondents testified that the design should be made at the waist to bring the balance with the other part. They mentioned that, small design on the waist line will work for this shape.

Respondent's views were solicited on the effect of smocking on the pear shape. The respondents' interviewed opined that smocking should be at the upper part since that area needs more weight to make the figure look well balanced and should be avoided at the lower part as it makes that area more bulky. They believe that designs on the bust line will enlarge this area to give balance to the other part of the figure. The respondents, upon further discussions, revealed that in order to broaden the upper part of the body, smocking should be concentrated on the upper section.

On the effects of Smocking on hourglass, the respondents indicated that, smocked decoration can be placed at either the upper or lower part of the body. They mentioned that if the design is placed at any part of the body and it will cause balance in the figure. Other 2 respondents believe that the design should be placed at the middle section to enhance the middle part of the body and again testified that heavy weight decorations will balance the figure when made at the waist.

\subsection{Effects of shirring on figure types}

Concerning the effects of shirring on apple shape, the respondents interviewed affirmed that, shirring can bring balance to the figure depending on how it is organised on the fabric, but should be avoided at the upper part. They continue to explain that placing the design at the wait will create illusion for the lower part making it a bit larger, but when placed at the upper body it will add volume to the area making the figure not balanced. The effect of shirring will be more pleasing at the top than the lower part.

The respondents were asked to tell the effects of shirring on the rectangular shape and they mentioned that, small shirring at the waist will enclose the waist. Shirring will better give a pleasing effect at the middle section than any part of the figure. The respondents further agreed that shirring gives a feminine shape when placed at the waist.

The views of the respondents were solicited on the effect of shirring as fabric decoration on pear shape. The respondents interviewed opined that shirring adds volume to the figure when placed at the upper part of the body and should be avoided at the hip area or lower part. They again mentioned that it will give good effect at the upper part of the body since it will bring out the buttocks and the hips of the end user.

On the issue of the effects of shirring on hourglass, it was found out that, shirring will be well pleasing at the upper section to give a well balanced figure and encourage viewers to focused on the rhyme at the upper section. Meanwhile, other respondents signify that depending on the position of the shirring it either can enlarge or enclose the figure as the figure is the ideal type. The respondents again said that when the design is placed at the middle part of the body it enhances and makes the shape more beautiful and make the shape more pronounced by bringing out the upper part as well as bringing out the hips and the buttocks. They added that, shirring at the waist line can enlarge the waist to create a balance on the figure type.

\section{Conclusion and Recommendation}

The study found that motifs and pattern decoration have effects on figure types The effect of fabric decorations (embroidery, appliqué, tie and dye and printing, smocking and shirring) on the female figure types (Apple shape, Straight/rectangular shape, Pear shape and Hourglass shape.) helps end users to know the effects of fabric 
decorations on their figure type and more or less enables the designers to select and design clothes for a particular figure type in terms of choice of fabric decoration. It may be concluded that perfect selection of fabric decoration for human body and all that goes with it enhances the individual's look. End users can improve their appearance with appropriate clothing by camouflaging their less desirable attributes and highlighting the more attractive aspects of their figure type. Furthermore, an understanding of how best a designer can decorate a fabric to make it more attractive on the end user is identifying the figure type of the client. Recognising the figure type of client enable the designer to determine where to place the decoration; either the upper part or the lower part to get a uniform figure.

It was recommended that periodic training sessions and refresher courses should be organised for the fashion designers to update them on fabric decoration and figure types of end users. This is mainly because most of the fashion designers choice for fabric decoration for various figure type is awful. Also, fashion designers should educate their clients or the ends users on how patterns and motifs decoration on the surface of fabrics affect their figure type. On the other hand, tailors, seamstresses and fashion designers should take figure types into consideration when selecting and producing garments for their clients or end users.

\section{References}

Ali, G., Eileen G. \& Maura, B. (2001), Through the Wardrobe Women's Relationship with their clothes. New York: Berg Oxford.

Almas F. M. (2018), Promoting Awareness about Traditional Motifs and Patterns of Pakistan in Truck Art through Textile and Fashion Design for the Local and Global Market. International Journal of Art and Art History, Vol. 6, No. 1, pp. 40-45 ISSN: 2374-2321

Amaden-Crawford, C. (2011), A guide to Fashion Sewing ( $5^{\text {th }}$ ed.). New York: Fairchild Books.

Arthur, P., Allen, C. C. \& Ingrid, J. (2005), Fabric Science $\left(8^{\text {th }}\right.$ ed.). New York: Fairchild publication, Inc.

Ashadown, S. P. \& O'Connell, E. K. (2006), Comparison of Test Protocols for judging the fit of mature womens apparel. Clothing and Textiles Research Journal. 24 (2).

Bonifant, S. (2009), Art and survival: A look at Textiles in the Gambia. Washington D.C: The Association of American Publishers

Caryn, F. (2011), How to Look Good Petite \& pear. Brilliant productions Ltd. Pg 52.

Colette, W. (1996), The Art of Manipulating fabric (2 ${ }^{\text {nd }}$ ed.). Ohio: Krause Publication.

Creswell, J. (2012), Educational research: Planning, conducting, and evaluating quantitative and qualitative research ( $4^{\text {th }}$ ed.).Upper Saddle River, NJ: Pearson Education.

Debiie S. (2013), Modern smocking Canadian smocking techniques and patterns, part I Vivebooks, and imprints of Rainbow Disk Ltd.

Durand, D. (1979), Smocking Technique, Project and Designs. New York: Courier Dover Publications.

Else, J. (1988). A composite of Indian Textiles Tradition and Technology, Arts Textring, Vol. X, Pg $71-84$.

Galleta, M. (2013), Embroidery Stitch Library. Toronto: Kiriki Press.

Hann, M. A. (2005), Patterns of culture techniques of Decoration and colouration. Pg. 29.

Hazel, K. (2001), Trends in Textiles Technology dyes and decoration. London: Read Educational and Professional Publishing Ltd.

Marjorie, M. (2007), UK Cooperative Extension Service CT - MMB.182. University of Kentucky-College of Agriculture. Extension Association for Textiles and Clothing, Pg 6

Picton, J. and Mack, J. (1991), African Textiles (2 $2^{\text {nd }}$ ed.). London: British Museum Publication.

Sorger R. \& Jenny, U. (2012). The Fundamentals of Fashion Design ( $2^{\text {nd }}$ ed.). USA: AVA Publishing SA.

Swati, B. (2010), Clothing and Textiles: A practical aspect. Rajasthan, India: Shree Niwas Publications.

Valerie, S. (2005), Encyclopaedia of Clothing and Fashion (Vol. 1). Boston: Thomson Corporation

Zheng, L. and Jin, K. (2014). Design of Decorative Fabrics in the Living Space of the Elderly. Studies in Sociology of Science. Vol. 5, No. 4, 2014, pp. 121-124 DOI: 10.3968/5943 